\title{
Enhanced odour-associated memory performance with a Y-maze assembly in Drosophila
}

Radhika Mohandasan ${ }^{1,2}$, Fathima Mukthar Iqbal ${ }^{1}$, Manikrao Thakare ${ }^{1,2}$, Madhav

Sridharan ${ }^{1,3,4}$, and Gaurav Das ${ }^{1 *}$

${ }^{1}$ Brain and Feeding Behaviour Laboratory, National Centre for Cell Science, S.P Pune University Campus, Ganeshkhind, Pune 411007, India

${ }^{2}$ Savitribai Phule Pune University, Ganeshkhind, Pune 411007, India

${ }^{3}$ Present address: Biozentrum, Department of Cell Biology, University of Basel, 4056

Basel, Switzerland and ${ }^{4}$ Friedrich Miescher Institute for Biomedical Research, 4058

Basel, Switzerland.

*Corresponding author - gauravdas@nccs.res.in 


\begin{abstract}
The neural basis of behaviour is identified by systematically disrupting the activity of specific neurons and screening for loss in phenotype. High scoring behavioural assays are thus necessary for identifying the neural circuits of novel behaviours. Here, we report the design and use of a Y-maze based classical olfactory learning and memory assay in Drosophila. Appetitive memory scores in our Y-maze are considerably greater and longer-lasting than that from a commonly used T-maze design. We show that testing in the $\mathrm{Y}$-maze is key to the improved scores. We also observed considerable 24-hour aversive taste reinforced memory performance with only one training trial using Y-mazes. This allowed us to determine the protein synthesis dependence of long-lasting aversive taste memories for the first time in flies. The Y-maze assembly will make olfactory conditioning more accessible and it will allow the study of novel memory phenotypes in Drosophila.
\end{abstract}

\title{
Keywords
}

Classical Pavlovian conditioning, olfactory conditioning, associative learning, long-term memory, LTM, Y-maze, T-maze, mushroom body, dopaminergic neurons, protein synthesis dependent 


\section{Introduction}

Behaviour emerges from the activity of neuronal networks in the nervous system. To identify the neural basis of a specific behaviour in flies, it is firstly reliably and robustly replicated in the laboratory. Second, specific brain regions or candidate neuronal subsets are methodically silenced by genetic means to look for change in the behaviour under study. This establishes a strong correlation of specific neural activity to behaviour that can be verified by further genetics, electrophysiology and functional imaging of neural activity (Simpson, 2009; White and Peabody, 2009; Griffith, 2012; Owald, Lin and Waddell, 2015; White, 2016). However, identification of neurons and neural mechanism underlying behaviour becomes difficult when performance scores are low and as a result, a meaningful reduction in behaviour becomes harder to observe. Higher performance scores are thus imperative. Any pronounced improvement over existing behaviour assays could also reveal novel behaviour phenotypes, allowing new lines of enquiry into the function of neural circuits.

In Drosophila, scientists have studied the neural mechanisms of learning and memory extensively for almost five decades (Keene and Waddell, 2007; Vosshall, 2007; Harris, 2008; Kahsai and Zars, 2011). The basic design of the Pavlovian associative learning assays, that has been central to this field, has remained unchanged. Groups of flies are sequentially exposed to two sensory stimuli, usually two odours, only one of which overlaps with either punishments like electric shock, heat and aversive taste or rewards like food, water, alcohol and sex (Pitman et al., 2009; Kahsai and Zars, 2011; Kaun et al., 2011; Shohat-Ophir et al., 2012; Das et al., 2014; Galili et al., 2014; Lin et al., 2014; Tabone Christopher and de Belle, 2014; Masek et al., 2015). Subsequently, the degree of avoidance or approach to the associated odour is quantified to derive a memory score via the calculation of a performance index.

In the early days of the field, mutagenesis screens for learning and memory-related genes allowed genetic inroads into memory mechanisms (McGuire, Deshazer and 
Davis, 2005; Skoulakis and Grammenoudi, 2006). Thereafter, the marked development of transgenic tools in Drosophila for manipulating the activity of specific neural subsets (Yoshihara and Ito, 2012; Owald, Lin and Waddell, 2015; Simpson and Looger, 2018), enabled detailed investigation of the neural circuitry underlying learning, memory-consolidation and recall (Keene and Waddell, 2007; Boto, Stahl and Tomchik, 2020). In the last decade or so, the focus has been on genetic refining of control over increasingly restricted neuronal populations (Pfeiffer et al., 2008, 2010; Jenett et al., 2012), electron microscopy-based circuit reconstruction (Zheng et al., 2018; Pipkin, 2020; Scheffer et al., 2020) and in vivo imaging of live fly brains (Simpson and Looger, 2018). This has now led to a richly complex picture of the neuronal connectivity and rationale that underlie learning and memory in flies (Cognigni, Felsenberg and Waddell, 2018; Boto, Stahl and Tomchik, 2020).

In the interim, olfactory conditioning assays have also been through developments of their own. The Benzer laboratory refined one of the earliest associative learning and memory experiments using the countercurrent apparatus to show that flies can learn to avoid odour associated with an electric shock (Quinn, Harris and Benzer, 1974). The same study described the use of a Y-maze to train flies to discriminate between two visible wavelengths of light (Quinn, Harris and Benzer, 1974). The authors used both the apparatus without active odour streams and the experiments yielded low learning scores. Later, although the countercurrent machine was used to condition flies with sugar or shock, learned odour preference was tested in a T-maze (Dudai, 1983; Tempel et al., 1983). Over the ensuing years T-maze design developed to accommodate both training and testing of flies and memory scores improved too (Tully and Quinn, 1985; Schwaerzel et al., 2003; Keene et al., 2006). Authors attributed this improvement to, a) shift from instrumental to classical conditioning where flies are unable to avoid the odours and reward/punishment, b) increased odour concentration and c) reduced 'distracting' stimuli like light (Tully and Quinn, 1985). This T-maze design or variants of it are now widely used for assaying learning and memory in flies (Y-C Kim, Lee and Han, 
2007; Colomb et al., 2009; Ichinose and Tanimoto, 2016). In contrast, Y-maze based designs have been relatively less utilised for memory assays in Drosophila. Current avatars have active odour streams and yield memory scores mostly comparable to the T-mazes (Kaun et al., 2011; Albin et al., 2015; Scaplen et al., 2020).

While effective, T-mazes can be cumbersome to work with and difficult to set up. It needs elaborate vacuum tubing arrangements, airflow, and pressure adjustments for consistent results. Commercially available T-mazes are expensive. Getting one made independently requires the services of a good machine shop. Importantly, both appetitive and aversive food component reinforced memory scores in a T-maze have room for improvement. Any new setup that addresses the above issues will be useful for existent users and those starting classical conditioning experiments in flies.

Here, we describe and benchmark a setup for olfactory conditioning of flies with food-component reinforcement. We trained flies in fly vials fitted with 3D printed odour cups. We tested conditioned odour preference of trained flies in Y-mazes put together from fly vials, 3D printed connectors and standard $1 \mathrm{~mL}$ micropipette tips. These assemblies are low cost, easy to assemble, customisable and do not require vacuum generated odour flows. The setups yield significantly higher and more persistent olfactory memory scores when compared to the widely used T-mazes. Our experiments suggest that better scores arise from testing in the $\mathrm{Y}$-mazes and do not depend on the training setup. We also obtained enhanced 24-hour aversive taste memory performance with copper sulphate $\left(\mathrm{CuSO}_{4}\right)$ reinforcement in our $\mathrm{Y}$-mazes. This allowed us to test, for the first time in flies, the protein synthesis dependence of long-lasting aversive gustatory memories. 


\section{Materials and Methods}

\section{Flies}

All flies were reared on food containing sugar, cornflour, malt extract and yeast at $25^{\circ} \mathrm{C}$ and $60 \%$ humidity, with a 12-12 hour light-dark cycle in incubators. We used previously described fly strains for all experiments: Wild type Canton-S (CS), dumb ${ }^{1}$ and dumb ${ }^{2}$ mutants (Young-Cho Kim, Lee and Han, 2007), R58E02-GAL4 (Liu et al., 2012) and UAS-shi ${ }^{\text {ts1 }}$ (Kitamoto, 2001).

\section{Chemicals and other materials}

White oil, a petroleum derived mineral oil, of ISO VG 12 grade (Shield Lubricants), with a specific gravity of $\sim 0.8\left(23^{\circ} \mathrm{C}\right)$ was procured locally. Sucrose (ANJ biomedicals-cat no. 57-50-1), octan-3-ol (SIGMA-Aldrich-cat No. 218405), 4-methyl cyclohexanol (SIGMA-Aldrich- cat no.153095), agar (HiMedia-cat no. GRM026), $\mathrm{CuSO}_{4} \cdot 5 \mathrm{H}_{2} \mathrm{O}$ (SIGMA-Aldrich cat No.- 209198), D-arabinose (MP Biomedicals- cat No.-194023), Whatman filter paper (GE-cat no. 3030-917) , Cycloheximide (MP Biomedicals- cat No.100183) and Polylactic acid (PLA) black 2.85 mm filament Ultimaker-cat No-1609) were procured from authorised vendors.

\section{Odour preparation}

For Y-maze conditioning $0.8 \mu$ l of octan- 3-ol (OCT) or $0.9 \mu$ l of 4-methyl cyclohexanol $(\mathrm{MCH})$ was diluted in $1.25 \mathrm{~mL}$ of white oil. We considered this as $\sim 10^{-3}$ or 1000 fold dilution. For odour standardisation experiments $10^{-3}$ dilutions were further serially diluted to $\sim 10^{-4}$ or $\sim 10^{-6}$. For T-maze conditioning, $\sim 10^{-3}$ odour concentrations were used (8-12 $\mu \mathrm{l}$ of OCT and $9 \mu \mathrm{MCH}$ in $10 \mathrm{~mL}$ white oil).

\section{Olfactory associative conditioning using Y-mazes}

\section{a. Training setup in fly vials.}

$5 \mathrm{ml}$ of $0.75 \%$ agar or sucrose (50 mM -to- $2 \mathrm{M})$ in $0.75 \%$ agar was poured in standard fly rearing vials (inner diameter: $23 \mathrm{~mm}$, outer diameter: $25 \mathrm{~mm}$, height: $90 \mathrm{~mm}$ ) and 
were used for appetitive training. For aversive taste training $80 \mathrm{mM} \mathrm{CuSO}_{4}+200 \mathrm{mM}$ arabinose in $0.75 \%$ agar was used. For training with dry sugar, $2.66 \mathrm{M}$ sucrose solution was poured and dried on pieces of filter paper $(\sim 8.5 \mathrm{~cm} * 7.3 \mathrm{~cm})$. The sugar papers lined the inner side of the training vials (Fig S2C).

$20 \mu \mathrm{l}$ odour dilutions were spotted on $1 \mathrm{~cm}$ * $1 \mathrm{~cm}$ Whatman filter paper pieces, which were placed inside custom-designed 3D printed odour cups with slotted base. The cups fit on top of the training vials (Fig 1A, B; Fig S1A, B and supplemental video file 1). The slots in the cup base allow diffusion of the odours into the tube below. We sealed the top of the odour cups with tape to prevent outward odour diffusion. Capped empty vials were used to harbour flies for the 5 minute in between their exposure to the two odours.

\section{b. Differential appetitive and aversive olfactory conditioning (training)}

4-8 days old mixed-sex population of flies were used for all experiments. We starved groups of $\sim 100$ flies in individual vials with $0.75 \%$ agar for $\sim 22-24$ hours at $25^{\circ} \mathrm{C}$. A small strip of filter paper was always provided inside the starvation vials as an additional surface for the flies to climb on. This kept flies from aggregating on the surface of the agar bed and getting their wings stuck wet. Before training we put 20-30 wild-type flies that were not being used for the experiment into each training vial for 20 minutes to imbue the vials with 'fly smell'. This was intended to reduce stress on the experimental flies later. The training tubes were also simultaneously allowed to odorize with the appropriate odour from the odour cups on top.

The conditioned stimuli (CS), odours OCT and $\mathrm{MCH}$, were reciprocally paired with only agar (CS- :- CS without unconditioned stimuli) or with sugar reward/aversive taste punishment in agar ( $\mathbf{C S}^{+}$:- CS with unconditioned stimuli). To begin, starved flies were transferred to the assigned agar vials to experience the $\mathrm{CS}^{-}$odour for 5 minutes. The flies were then transferred to empty vials for 5 minutes. Next, they were carried on to the $\mathrm{CS}^{+}$odour vial with either sugar-agar reward or aversive substance-agar 
punishment for 5 minutes. Any change in association times during training are mentioned where appropriate. Food dyes were added to the CS+ agar to visually monitor feeding during standardization of conditioning protocols. Training vials were inverted during conditioning with aversive substances and during conditioning with shorter association time (2 minutes) to ensure that hungry flies find the agar bed readily due to their negative geotactic tendencies. After training, flies were maintained in 0.75 $\%$ agar vials to keep them hungry until testing 24 hours later. For assaying 3, 5 or 7 day memory, flies were starved for $\sim 6$ hours after training in $0.75 \%$ agar vials.

Subsequently, they were transferred to food vials until 24 hours before planned testing time. They were then shifted back to $0.75 \%$ agar vials for $\sim 24$ hour starvation until testing.

For blocking specific neuronal populations during training using the UAS-Shibire ${ }^{\text {ts } 1}$ transgene (Kitamoto, 2001), flies in starvation vials were kept at $32^{\circ} \mathrm{C}$ in a heat chamber specially designed for behavioural experiments for 30 minutes before training (Fig S1E). The training sequence was also carried out at $32^{\circ} \mathrm{C}$ inside the chamber. After training, flies were shifted back to room temperature $\left(22-23^{\circ} \mathrm{C}\right)$ and eventually at $25^{\circ} \mathrm{C}$ for storage until testing.

\section{c.Y-maze testing setup}

The 3D printed parts of the $Y$-maze were designed on the free online 3D design software TINKERCAD and printed on ULTIMAKER 2 PLUS using PLA filaments. These parts include the central Y-connector with three arms, 3 vial connectors, and a fly loading vial (Fig 1A and S1A). The STL files for all 3D printed parts, including those for training, are available upon request to the corresponding author.

The odour choice vials are the same type as the standard fly-rearing vials used for training. Whatman filter paper pieces $\left(2.5 \mathrm{~cm}{ }^{*} 2 \mathrm{~cm}\right)$ were spotted with $20 \mu$ of either $\mathrm{CS}^{-}$or $\mathrm{CS}^{+}$odour dilutions and were wedged in at the bottom of the vials. The broader 
end of an individual vial connector was attached to each of the two odour vials, with a 1 $\mathrm{mL}$ end cut tip fixed into the connector, facing inwards into the vials. The narrow end of a vial connector fitted to individual arms of the central $Y$ connector. The central $Y$ connector has 3 equal length arms that are 120 degrees apart from each other. Snug-fitting at all joints, namely those between the cut tip to vial connector, vial to vial connector, vial connectors to $Y$ connector is ensured by wrapping masking tape onto the appropriate surfaces (Fig S1C). The third arm of the $\mathrm{Y}$ connector is connected to the opaque (3D printed) fly loading vial. The odour choice vials are wrapped in black tape to prevent the influence of non-uniform lighting on choice. They can be left uncovered if uniform lighting is ensured to both the choice arms during testing.

\section{d. Conditioned odour preference of flies in Y-mazes (testing)}

Before the start of testing, the odour laden filter papers were placed in the appropriate vials and the $\mathrm{Y}$-mazes were assembled as described. They were left to odorize for 20 minutes. Because there are no vacuum-aided odour flows created in the Y-maze, it is important to carry out testing at fixed times after odours are aliquoted and Y-mazes assembled. This is because the strength of the volatile odours tends to change over time and influence choice.

Previously conditioned starved flies were loaded onto the Y-mazes using the 3D printed opaque vial. After loading, the Y-mazes were kept upright in a plastic rack (Figure S1D, E). The experiments should ideally be carried out in stable temperature and uniform light settings. As previously mentioned, we use a custom-designed chamber for testing (Figure S1E). The negative geotactic instinct of flies ensured that they climb up through the $Y$ connector and move towards either one of the odour choice vials. Once individual exploring flies have walked through the cut $1 \mathrm{ml}$ pipette tip into one of the odour vials, they were effectively trapped as they were unable to crawl back into the tip. We generally used a testing time of 30 minutes. As assay scores are determined by trapping the flies, the $\mathrm{Y}$-mazes can be left for a longer period of time for testing. Testing 
can also be done with the Y-mazes laid horizontally. In such cases, we suggest that the choice vials are left uncovered to experience uniform light. After testing the $\mathrm{Y}$-mazes were transferred to a freezer for at least 45 minutes to freeze the flies and then their number in each odour vial was counted manually. A memory-driven odour preference score or performance index (PI) was calculated as follows:

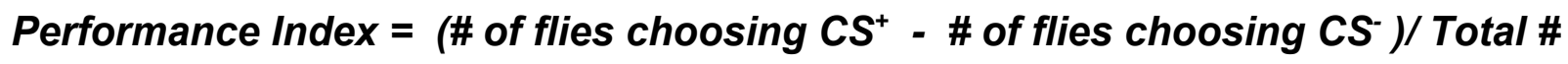
of flies that made choice

To prevent skewed memory scores due to odour bias, half of the fly groups for each condition or genotype is trained with OCT as $\mathrm{CS}^{+}$and the other half with $\mathrm{MCH}$ as $\mathrm{CS}^{+}$. Each group of flies, conditioned with either one of the odours is considered as ' $n=1$ '. To reduce variability, scores from any $\mathrm{Y}$-maze where less than 30 flies had made the choice were not included in the final analysis.

\section{Odour balancing in Y-mazes}

Memory score is determined by the distribution of a group of conditioned flies between the two odour choice vials (see above). Therefore, establishing odour balance at which naive or unconditioned flies show an equal preference for $\mathrm{OCT}$ and $\mathrm{MCH}$, is critical before using the $\mathrm{Y}$-mazes for training. For odour balancing, the $\mathrm{Y}$-maze is prepared with concentrations of $\mathrm{OCT}$ and $\mathrm{MCH}$ that are presumed to be equally repulsive/attractive to flies naively. $\sim 100$ naive flies are starved for $\sim 22-24$ hours on $0.75 \%$ agar and their choice determined as described in the previous section. A preference index of odour choice is calculated as:

\section{Preference Index = (\# of flies choosing OCT- \# of flies choosing MCH /Total number of flies that made a choice)}

Hence a preference index of +1 denotes an absolute OCT preference and -1 denotes an absolute preference for $\mathrm{MCH}$. If the starting odour concentrations yield a skewed distribution, the concentration of the avoided odour can be lowered or the concentration of the preferred odour increased until an average equal preference is achieved. 
Important to note here is that odour balances vary with temperature and should be empirically determined as needed.

\section{Olfactory associative conditioning using T-mazes}

T-maze conditioning protocols have been extensively described (Pitman et al., 2009;

Michael J. Krashes and Waddell, 2011; M. J. Krashes and Waddell, 2011). Following is a brief description.

\section{a. T-maze setup}

The T-maze is made of three pieces of acrylic; two side walls screwed to a metal base and a middle piece, with an elevator cavity, sandwiched in between the side walls with clamps (Fig S1F). It has an upper training zone and a lower testing zone that allows binary choice. A single tube connected to an odour source can be fixed at the training zone. The testing zone has two $180^{\circ}$ opposing tubes each connected to either one of the odour sources. The elevator in the middle allows the transport of flies from the training to the testing zone. The training and testing zones are connected to a vacuum pump which pulls the training and testing odour streams through the tubes. Constant air pressure was maintained by clamping the T-mazes. Air pressure was frequently checked and adjusted as needed during an experiment using an airflow meter.

\section{b. T-maze training and testing}

Flies starved for 24 hours on $0.75 \%$ agar were trained in the upper training tube of a T-maze to pair CS- odour with $0.75 \%$ agar poured on a Whatman filter paper $\left(4.8 \mathrm{~cm}^{\text {* }}\right.$ $7.5 \mathrm{~cm}$ ) or dry filter paper for 2 minutes. $\mathrm{CS}^{-}$odour source was then disconnected to have only room airflow through the tube for 30 seconds. Following this the flies were transferred to the $\mathrm{CS}^{+}$odour tube, lined with a filter paper with a layer of $2 \mathrm{M}$ sucrose (or appropriate sugar/bitter US) in $0.75 \%$ agar, for 2 minutes. Any variation in association times is noted in the appropriate places. 
After training, flies were transferred to $0.75 \%$ agar for 24 hours until testing. In some protocols, these are clearly mentioned in relevant places, they were fed briefly $\sim 6$ hours after training to ensure that enough flies survive for testing. For testing, flies were loaded onto the T-maze in a tube via the upper training zone and lowered to the testing zone, where they are given 2 minutes under uniformly illuminated conditions inside behaviour chambers to choose between the opposing $\mathrm{CS}^{+}$and $\mathrm{CS}^{-}$odour streams. Elevator is raised after 2 minutes, trapping the flies in the individual odour tubes. The flies from these tubes are then transferred to separate vials and subsequently frozen. The number of flies choosing each odour is counted manually. A memory-driven performance index $(\mathrm{PI})$ is calculated the same as with $\mathrm{Y}$-mazes:

Performance Index = (\# No. of flies choosing CS ${ }^{+}$- \# No. of flies choosing CS )/ Total \# of flies that made a choice

As with Y-mazes, we trained an equal number of groups with OCT and $\mathrm{MCH}$ as the $\mathrm{CS}^{+}$ odour as much as possible. We considered each group of flies, conditioned with either one of the odours as $n=1$.

\section{Cycloheximide feeding}

As described before, $\sim 35 \mathrm{mM}$ of cycloheximide was mixed into $0.75 \%$ agar when flies were being starved before training for 16 hours (Tully et al., 1994; Krashes and Waddell, 2008). Cycloheximide was dissolved in warm water and subsequently mixed into melted agar before it was poured and solidified in the starvation vials.

\section{Data plotting and Statistical analysis}

Statistical analysis was performed using the software GraphPad Prism version 8.3.1.

All data points are represented as a scatter plot in which each point represents a single 'n' with mean and \pm standard deviation (SD). P values for relevant comparison of groups for all data and other relevant statistical details are reported in Supplemental table 1 (ST1). 
We plotted our data as estimation plots. These plots include a representation of the 'effect' size, which here is the difference in means of the compared groups $(\Delta$ Performance Index- $\Delta \mathrm{PI}$ ). While the data itself were plotted using GraphPad Prism, we used the DABEST ('data analysis with bootstrap-coupled estimation') web application at https://www.estimationstats.com for generating the effect size plots (Ho et al., 2019). The $\Delta \mathrm{PI}$ or the effect size axis, placed to the right of the main graphs, has its origin at the control group mean. The effect size is aligned with the experimental group mean on this axis (large black dot). The curve represents bootstrap sampling error distribution of $\Delta \mathrm{PI}$. The $95 \%$ Confidence Interval $(\mathrm{Cl})$ of the effect size is depicted by the two ends of the vertical error bars intersecting the effect size. The software generates the $95 \% \mathrm{Cl}$ and the error curve by performing 5000 bootstrap (with replacement) resampling of the groups being compared and computing the difference in means of the resampled groups. The software also applies bias-corrected and accelerated (BCa) correction to the resampling bootstrap distributions of the effect size, to account for any skew in it. Further details can be found at https://www.estimationstats.com. 


\section{Results}

Training in vials and testing in Y-mazes is able to assay robust long-term memory

To simplify the handling and assembly of olfactory conditioning setup in flies, we adapted a Y-maze based design without active (vacuum-assisted) odour streams (Fig 1A, Fig S1A-E). Design, build and details of olfactory conditioning and odour preference testing are described in the Materials and Methods section.

We started by training groups of flies with $2 \mathrm{M}$ sucrose reinforcement and tested for 24-hour memory performance, with $10^{-3}, 10^{-4}$ and $10^{-6}$ dilutions of $\mathrm{OCT}$ and $\mathrm{MCH}$, for both training and testing (Fig 1B). Naive/untrained flies were found to prefer the two odours equally at the above concentrations (Fig S2A). We observed robust performance with $10^{-3}$, drastically lowered but intermediate performance with $10^{-4}$ and no discernible performance with $10^{-6}$ odour concentrations (Fig 1B). Based on our results, we decided to train and test flies with $10^{-3}$ dilutions of $\mathrm{OCT}$ and $\mathrm{MCH}$ for all subsequent experiments.

In all above cases flies had 30 minutes to choose between the two odour choice vials as this proved to be a sufficient period to allow most flies to make a choice (Figure S2B). It is important to note here that we observed that a large number of flies are trapped inside the choice vials by $\sim 10-15$ minutes (data not shown). But we decided to go with 30 minutes for convenience of use, and to ensure a majority of flies have made a choice. We did not go for a longer choice time to prevent the choice being affected by an altered odour balance.

We next examined the effect of $\mathrm{CS}^{+}$association time on memory performance. Decreasing odour with $2 \mathrm{M}$ sucrose association time down to 2 minutes from 5 minutes did not affect performance (Fig 1C). However, flies trained for 5 minutes with sucrose showed lower mortality upon subsequent starvation (data not shown). For most experiments, we subsequently preferred to use the 5 minute association time due to the 
lower mortality rate and also because the 5-5-5 minute (CS-no odour-CS ${ }^{+}$) training regimen conveniently facilitated the conditioning of multiple groups of flies simultaneously.

We also investigated the effect of training with different concentrations of sucrose on 24-hour memory scores. We observed considerably reduced memory scores when flies were trained with $100 \mathrm{mM}$ or $50 \mathrm{mM}$ sucrose compared to with $2 \mathrm{M}$ sucrose training (Fig 1D). Since training with $2 \mathrm{M}$ sucrose facilitated the highest 24-hour memory performance, we used this concentration of sucrose for further experiments. We also trained flies with 2.66 M saturated sucrose solution poured and dried on paper, which lined the training vials. Here, the scores were robust too, but reduced compared to training with $2 \mathrm{M}$ Sucrose in agar for the same association time of 2 minutes (Fig S2C, Fig 1C). Next, we tested how long memory lasted in our setup. For this, we tested $2 \mathrm{M}$ sucrose (in agar) reinforced memory performance 1, 3, 5 and 7 days after training. We found that despite expected decay of memory score over time, 7-day memory performance was robust (Fig 1E). 


\section{Figure 1}

bioRxiv preprint doi: https://doi.org/10.1101/2020.11.18.386128; this version posted November 20, 2020. The copyright holder for this preprint

A

(which was not certified by peer review) is the author/funder, who granted bioRxiv a license to display the preprint in perpetuity. It is made available under aCC-BNAC-ND 4.0 International license.
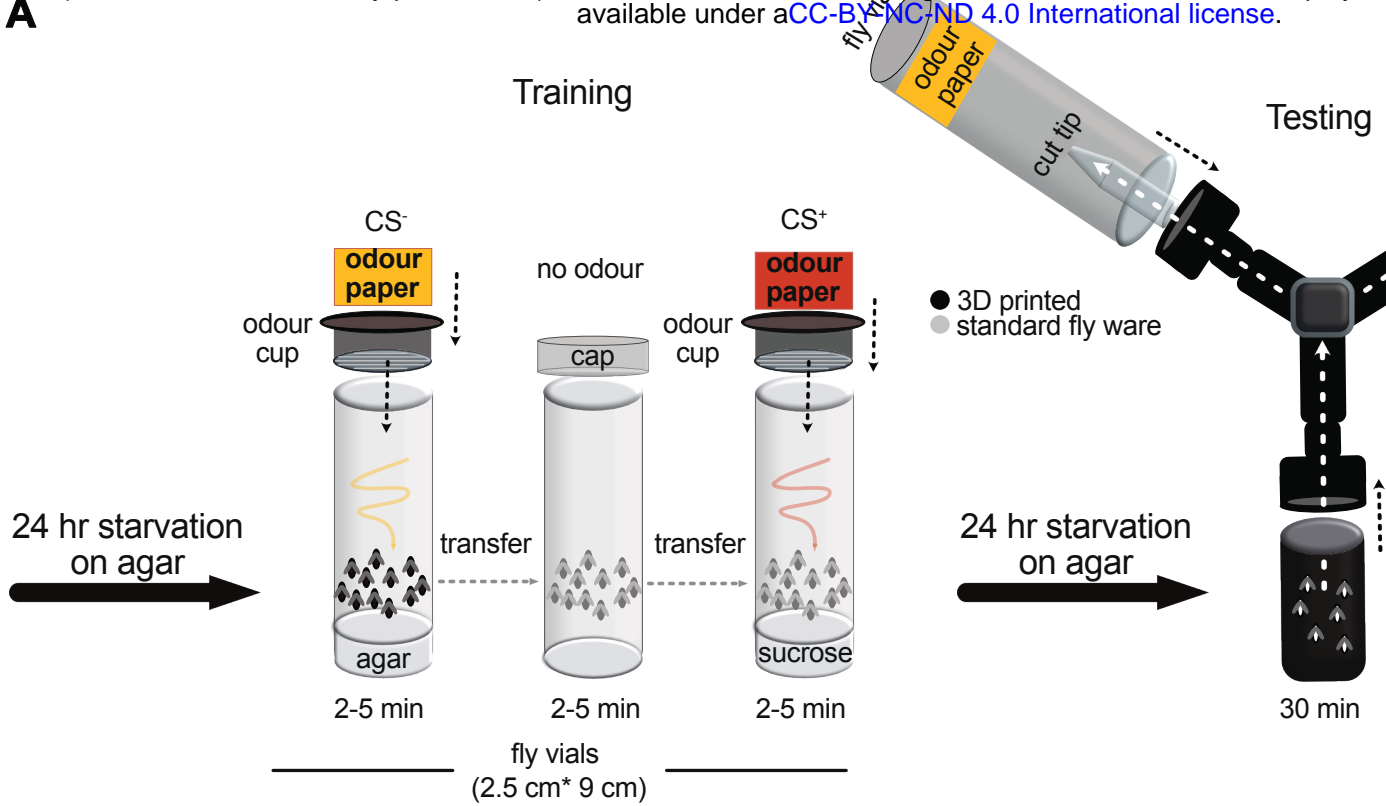

standard fly ware

B

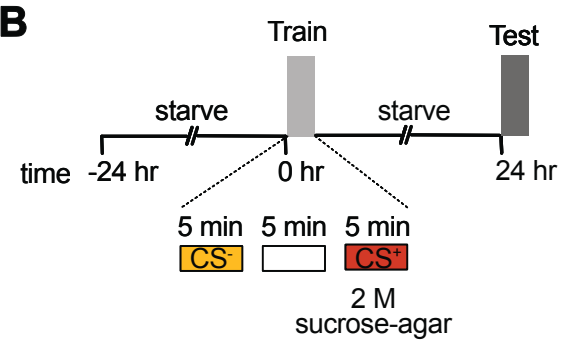

odour dilutions used

for training and testing

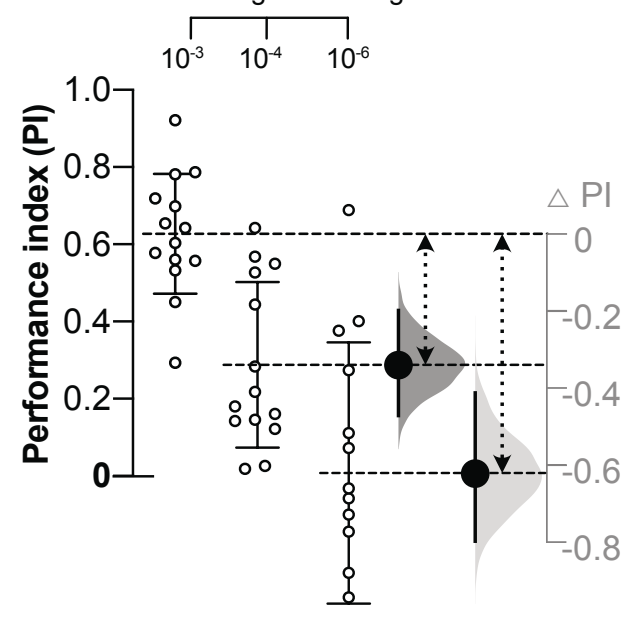

$:$

$\mathbf{E}$

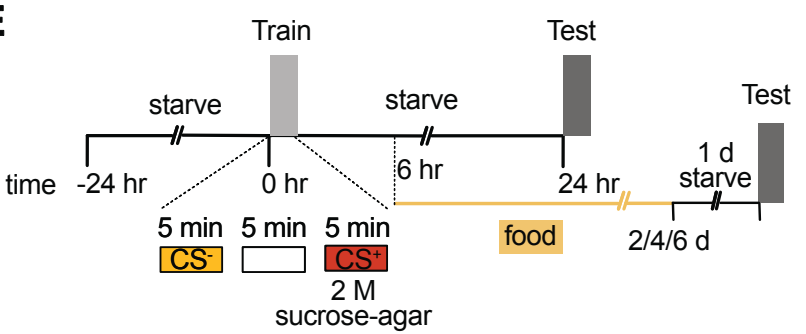

C Train Test

D

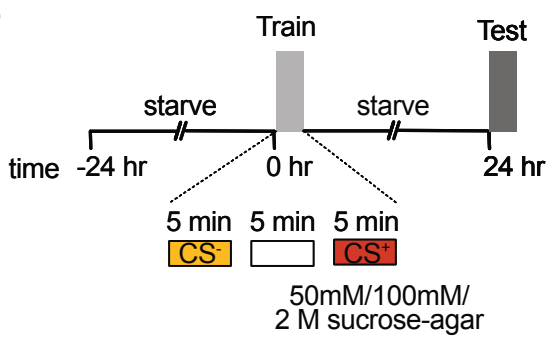

sucrose concentrations

association time during training

5 min 2 min

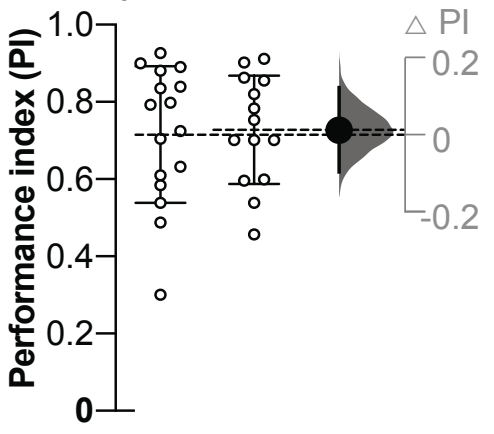

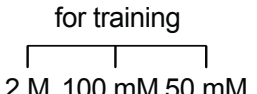

$2 \mathrm{M} 100 \mathrm{mM} 50 \mathrm{mM}$

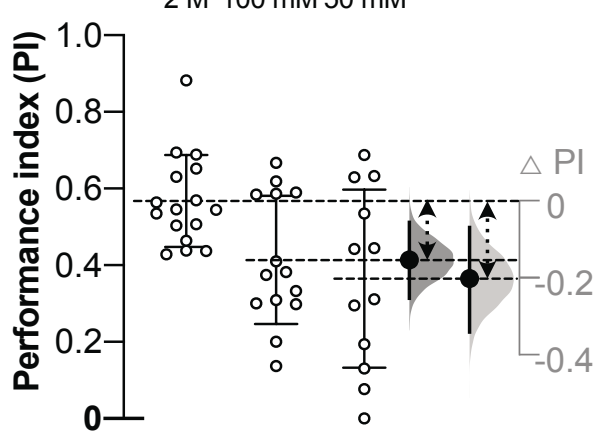

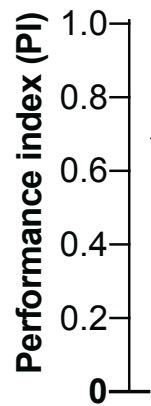

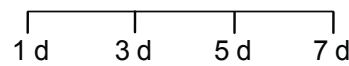

$\circ \stackrel{\circ}{\circ} \circ 0 \circ 0$

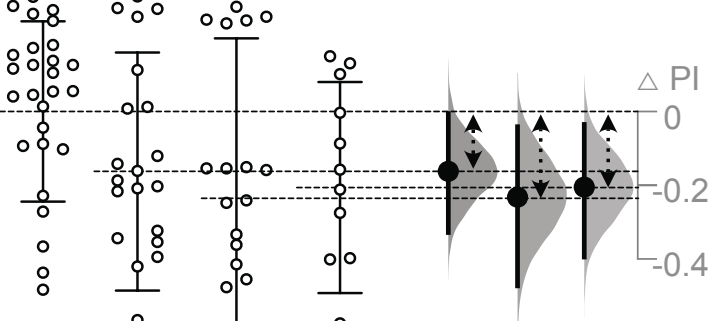




\section{Figure 1: Robust and long-lasting memory performance using Y-mazes: (A)}

Protocol for classical odour conditioning of flies in vials and subsequent testing of conditioned odour preference in Y-mazes without vacuum-assisted active airflows. The setups are built with a combination of readily available fly plasticware and custom-designed 3D printed parts. (B) Using $\sim 10^{-3}$ fold dilution of OCT and $\mathrm{MCH}$ for both training and testing yielded the maximum score, compared to $\sim 10^{-4}$ or $\sim 10^{-6}$ dilutions , $n=14 ; C S^{+} O C T=7, M C H=7$ for all groups. (C) Varying odour and $2 \mathrm{M}$ sucrose association time from 5 minutes $\left(n=16 ; C^{+} O C T=8, M C H=8\right)$ to 2 minutes $\left(n=14 ; C S^{+}\right.$ $\mathrm{OCT}=7, \mathrm{MCH}=7$ ) during training, did not change 24-hour memory performance. (D) Lowering sucrose concentration used for training from $2 \mathrm{M}\left(n=16 ; \mathrm{CS}^{+} \mathrm{OCT}=8, \mathrm{MCH}=8\right)$ to $100 \mathrm{mM}\left(n=14 ; C S^{+} O C T=7, M C H=7\right)$ and $50 \mathrm{mM}\left(n=12 ; C S^{+} O C T=6, M C H=6\right)$ greatly reduced 24-hour memory performance. (E) $2 \mathrm{M}$ sucrose reinforced memory performance with Y-mazes at 1 day $\left(n=28 ; C S^{+} O C T=14, M C H=14\right), 3$ day $(n=21$; $\left.C S^{+} O C T=11, M C H=10\right), 5$ day $\left(n=18 ; C S^{+} O C T=10, M C H=8\right)$ and 7 day $(n=12$; $\mathrm{CS}^{+} \mathrm{OCT}=6, \mathrm{MCH}=6$ ) after conditioning. Substantial $2 \mathrm{M}$ sucrose reinforced memory performance is seen even at the 7-day timepoint. To the right of each graph, effect size or the difference in mean performance index $(\Delta \mathrm{PI})$ plots are shown. The curve represents the bootstrap sampling error distribution of $\Delta \mathrm{PI}$. The observed effect size between experimental and a control group is depicted as a large black dot and the $95 \%$ $\mathrm{Cl}$ is indicated by the ends of the vertical error bars. Double-headed dashed arrows between the group means indicate the groups being compared. Also, see supplementary Figure S1 and S2. 


\section{Memory formation in our setup is dopamine signalling and PAM cluster activity dependent}

Olfactory appetitive memory formation in a T-maze requires dopamine release from the rewarding dopaminergic neurons of the protocerebral anterior medial (PAM) cluster and the dopaminergic receptor DopR1 in the mushroom body neurons (Young-Cho Kim, Lee and Han, 2007; Burke et al., 2012; Liu et al., 2012). To test whether appetitive memories formed in our setup were also dopamine signalling dependent, we first tested $2 \mathrm{M}$ sucrose reinforced 24-hour memory performance in two strains that are null mutants for the dopamine receptor DopR1, namely Dumb ${ }^{1}$ and Dumb ${ }^{2}$, along with wild type CS control flies (Young-Cho Kim, Lee and Han, 2007). Olfactory memory performance was almost completely abolished in the Dumb ${ }^{1}$ and Dumb $b^{2}$ mutants compared to the robust performance in the control (Fig 2A).

Next, we wanted to test whether the rewarding PAM dopaminergic neurons were required for appetitive memory formation during training in our setup. For this, we

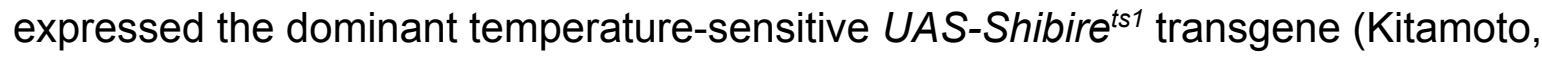
2001) in almost all PAM neurons using the R58E02-GAL4 driver line (Liu et al., 2012; Huetteroth et al., 2015; Yamagata et al., 2015). This allowed us to transiently silence the rewarding PAM dopaminergic neurons at the restrictive temperature of $32^{\circ} \mathrm{C}$ during training with $2 \mathrm{M}$ sucrose reinforcement. Upon testing memory performance at 24-hour, we saw that while robust memory performance is seen in the parental controls, the experimental flies, expressing UAS-Shibire ${ }^{t s 1}$ in R58E02-GAL4 labelled neurons, exhibited no apparent memory performance (Fig 2B). Importantly, such memory defects were not observed in the same genotypes when the experiment was performed at the permissive temperature of $24^{\circ} \mathrm{C}$ (Fig. 2C). Together, the above results ascertained that learning in our assay setup required dopamine release from the PAM neurons and also required the function of the DopR1 dopamine receptor expressed in the mushroom body neurons. 


\section{Figure 2}
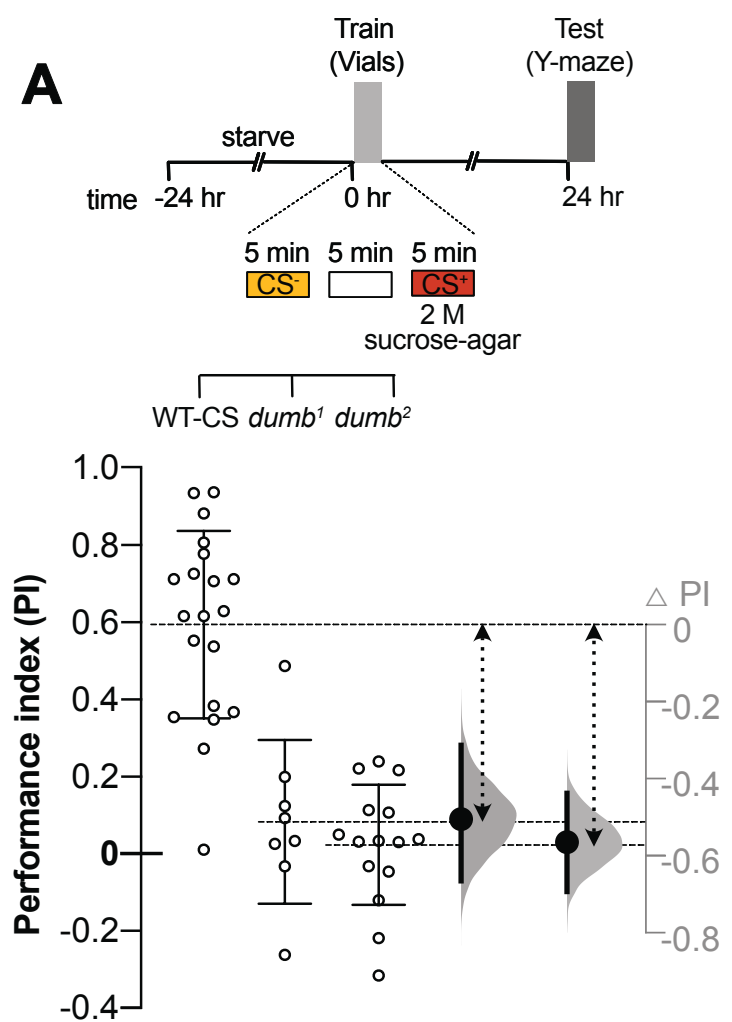

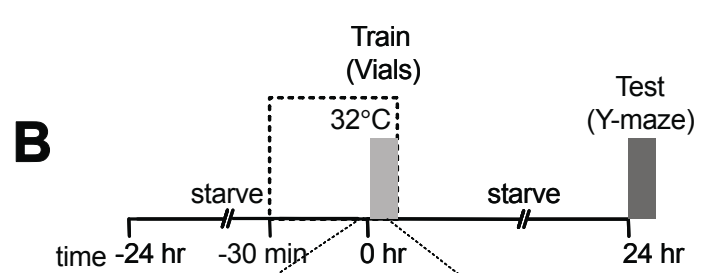

$5 \min 5 \mathrm{~min} 5 \mathrm{~min}$

$\mathrm{CS}^{\square} \square \frac{\mathrm{CS}^{+}}{2 \mathrm{M}}$

sucrose-aga
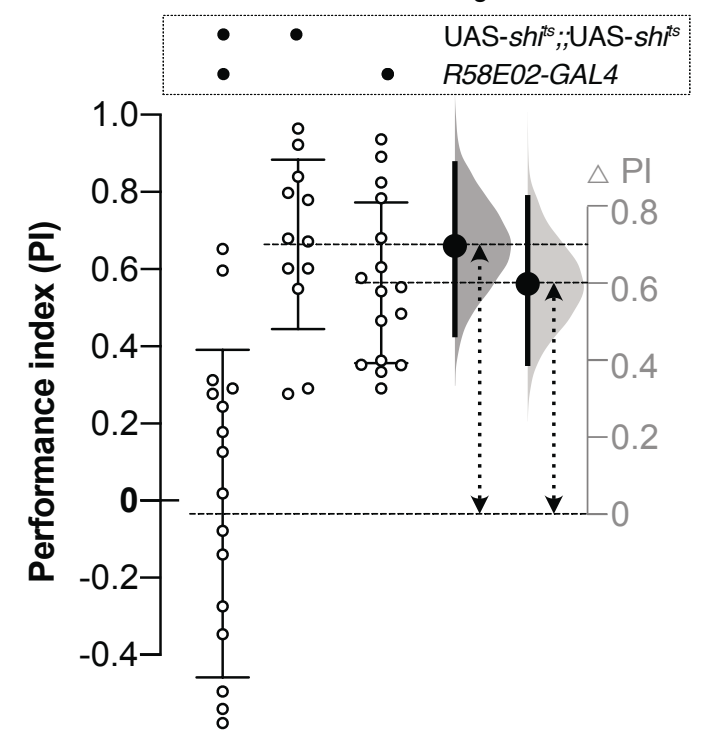
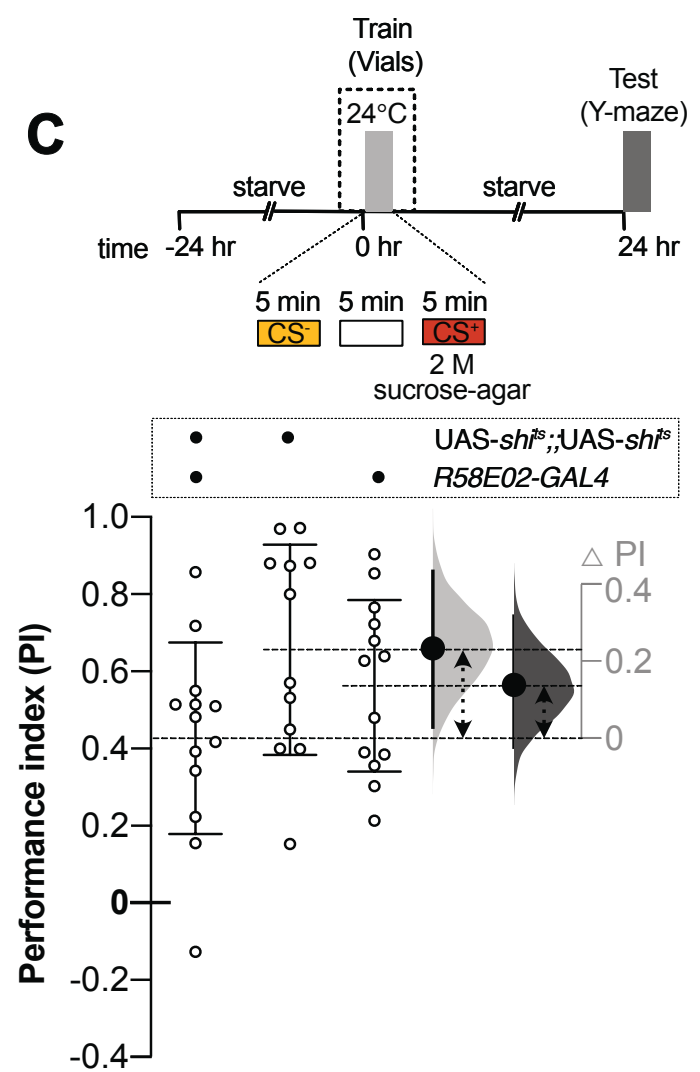
Figure 2: Appetitive memory formation is dopamine signalling dependent: (A) 24-hour Memory formation is DopR1 dopamine receptor-dependent. Compared to wild type CS flies ( $n=20 ; C S^{+} O C T=10, M C H=10$ ), appetitive memory performance is abolished in dumb ${ }^{1}\left(n=8 ; C S^{+} O C T=5, M C H=3\right)$ and dumb ${ }^{2}\left(n=15 ; C S^{+} O C T=7, M C H=8\right)$ mutants. (B) Activity of the reward dopaminergic neuron in the PAM cluster is required for appetitive memory formation during training in vials. Flies were trained with $2 \mathrm{M}$ sucrose reinforcement at $32^{\circ} \mathrm{C}$ to block PAM dopaminergic neurons during training. R58E02-GAL4 drove UAS-Shibire ${ }^{\text {ts } 1}$ transgene in reward PAM dopaminergic neurons ( $\left.n=17 ; C S^{+} O C T=9, M C H=8\right)$. Memory performance was severely impaired in these flies compared to the two parental lines, UAS-Shibire ${ }^{t s 1}\left(n=12 ; C S^{+} O C T=6, C S^{+} M C H=6\right)$ and R58E02-GAL4 ( $n=16 ; \mathrm{CS}^{+} \mathrm{OCT}=8, \mathrm{CS}^{+} \mathrm{MCH}=8$ ). (C) Room temperature control experiment for (B) shows that the experimental genotype, R58E02-GAL4/UAS-Shibire ${ }^{t s 1}$ ( $n=13 ; \mathrm{CS}^{+} \mathrm{OCT}=7 ; \mathrm{CS}^{+} \mathrm{MCH}=6$ ) performs as well as the parental controls UAS-Shibire ${ }^{t s 1}\left(n=12 ; C S^{+} O C T=7, C S^{+} M C H=5\right)$ and R58E02-GAL4 $\left(n=13 ; C S^{+} O C T=7\right.$, $\left.\mathrm{CS}^{+} \mathrm{MCH}=6\right)$. Effect size or the difference in mean performance index $(\triangle \mathrm{PI})$ plots are shown for all. They show the observed effect size of the experimental group compared to a control (large black dots), $95 \% \mathrm{Cl}$ (end of vertical error bars) and the bootstrap resampling error (curve) of effect size. Double-headed dashed arrows mark the groups being compared. 


\section{Testing in the Y-mazes is key to improved scores over the T-maze}

While our average $\mathrm{Y}$-maze performance indices for 24-hour memory are consistently higher ( 0.6) than reported using the T-maze, we wanted to do a side by side comparison with a frequently used T-maze paradigm (Tully et al., 1994; Michael J. Krashes and Waddell, 2011; M. J. Krashes and Waddell, 2011). For this, we trained groups of flies with $1 \mathrm{M}$ sucrose reinforcement in vials or in T-maze tubes for the same $\mathrm{CS}^{+}$association time of 2 minute. From both training conditions, half of the groups were tested in Y-mazes and the other half in the T-mazes for 24-hour memory. Hence, we had 4 groups in each experiment; a) vial-trained and Y-maze tested (VY), b) vial-trained and T-maze tested (VT), c) T-maze tube trained and Y-maze tested (TY) and d) T-maze tube trained and T-maze tested (TT) (Fig 3A, schema).

We observed that regardless of the training condition, in vials or in T-maze tubes, the groups tested in the $\mathrm{Y}$-mazes showed consistently greater mean performance than the groups tested in the T-mazes (VY > VT and TY>TT). This strongly suggested that testing in the Y-maze assembly was critical for the higher scores obtained. Also, groups tested in the same apparatus had similar scores regardless of the training conditions

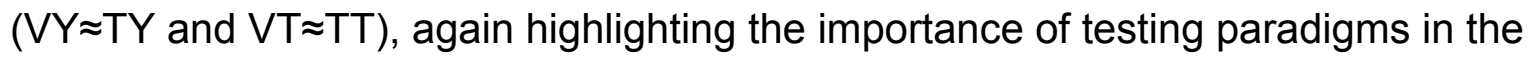
magnitude of memory performance (Fig $3 A$ ).

Next, we directly compared the longevity of memory performance using Y-mazes and $\mathrm{T}$-mazes. Fly groups were trained in vials and T-maze tubes with $2 \mathrm{M}$ sucrose reinforcement for 5 minute $\mathrm{CS}^{+}$association time. The memory performance of both these groups were tested 1 day and 5 days after training with the vial trained flies tested in Y-mazes (VY) and the T-maze tube trained flies tested in T-mazes (TT).

We found that, as before (Fig.1D), robust 1-day and 5-day memory performance could be seen in VY groups. At both time points, TT groups showed considerably reduced 
memory scores compared to the VY groups. However, the reduction was more pronounced on the 5th day, with a markedly weakened TT group mean score (Fig. 3B).

This suggested that workable memory scores were hard to derive with the T-mazes beyond 5 days, whereas robust 7 -day memory performance was clearly attainable with Y-mazes (Fig 1D). Therefore, clearly, the Y-maze assemblies yielded superior scores over a longer time too. 


\section{Figure 3}

bioRxiv preprint doi: https://doi.org/10.1101/2020.11.18.386128; this version posted November 20, 2020. The copyright holder for this preprint (which was not certified by peer review) is the author/funder, who has granted bioRxiv a license to display the preprint in perpetuity. It is made A
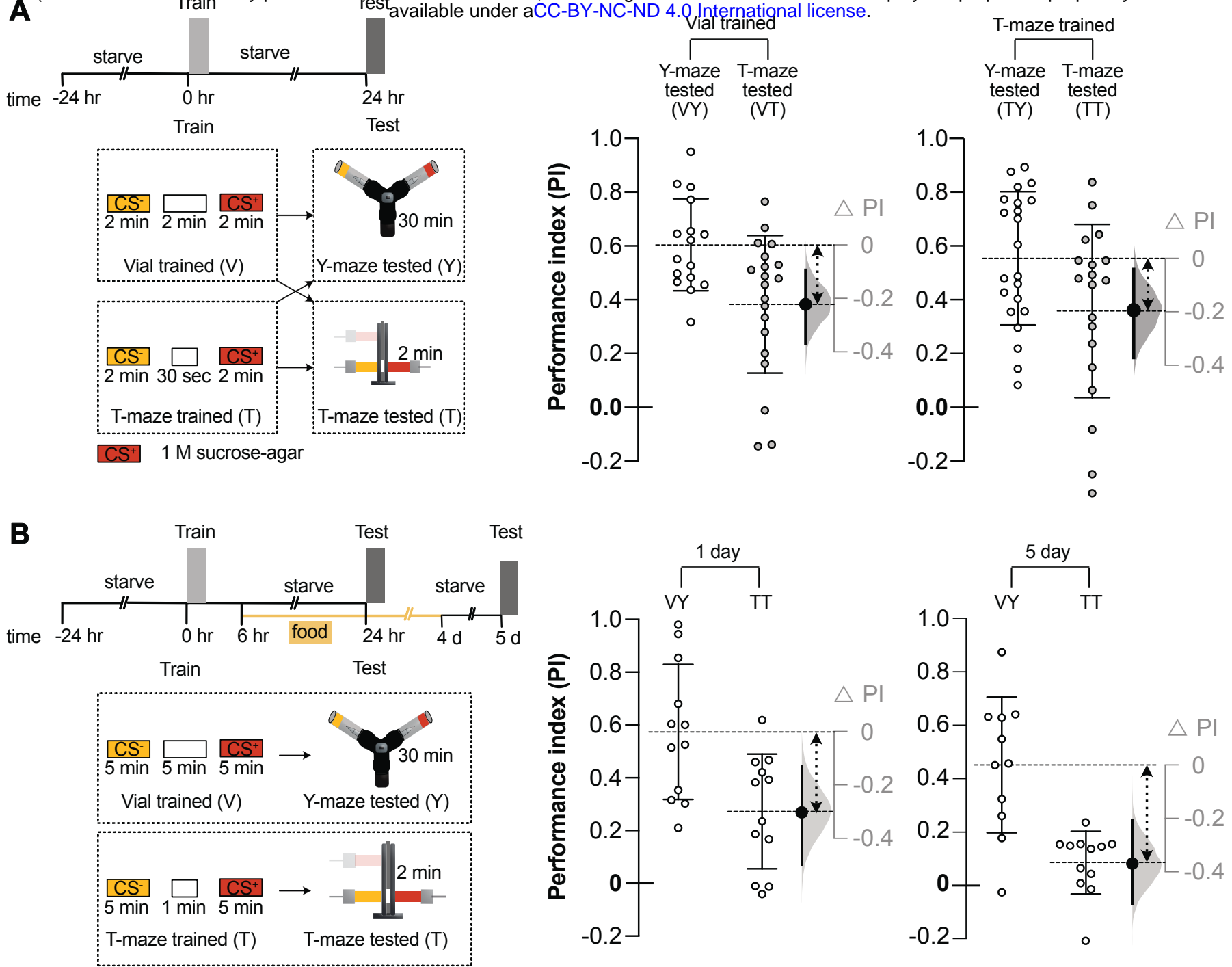

$\mathrm{CS}^{+} 2$ M sucrose-agar 
Figure 3: Olfactory memory assay using Y-mazes markedly improves scores over T-maze based assaying. (A) Direct comparison of training and testing in $\mathrm{Y}$-mazes and T-mazes. Results suggest that irrespective of training condition, in vials or in T-maze tubes, testing in $\mathrm{Y}$-mazes yields superior scores to that of the T-mazes. The Vial trained and T-maze tested group (VT; $n=20 ; C S^{+} O C T=11, M C H=9$ ) has considerably reduced performance from the Vial trained and Y-maze tested group (VY; $n=16 ; C S^{+} O C T=8$, $\mathrm{MCH}=8$ ). Similarly, the T-maze tube trained and T-maze tested group (TT; $n=19 ; \mathrm{CS}^{+}$ $O C T=9, M C H=10$ ) also has reduced performance from the T-maze tube trained and Y-maze tested group (TY; $n=22 ; C S^{+} O C T=11, M C H=11$ ). (B) 1 day and 5 day memory performance comparison between Y-mazes and T-mazes. Significant 1 day scores are seen in both the Vial trained and Y-maze tested group (VY) and the T-maze tube trained and T-maze tested group (TT). However, VY group (1 day; $n=12 ; C S^{+} O C T=6$, $\mathrm{MCH}=6$ ) has substantially higher memory performance than the TT group ( 1 day; $n=12$; $\left.\mathrm{CS}^{+} \mathrm{OCT}=6, \mathrm{MCH}=6\right)$. Similar trend is seen on day 5 for the two groups. However, while VY (5 day; $n=11$; $C S^{+} O C T=5, M C H=6$ ) performance is still robust, TT group ( 5 day; $n=12$; $C S^{+} O C T=6, M C H=6$ ) performance is close to zero. Effect size or the difference in mean performance index $(\Delta \mathrm{PI})$ plots are shown for all. They show the observed effect size of the experimental group compared to a control (large black dots), $95 \% \mathrm{Cl}$ (end of vertical error bars) and the bootstrap resampling error (curve) of effect size.

Double-headed dashed arrows mark the groups being compared. 


\section{4-hour sweet and aversive taste memories are protein synthesis-dependent}

Previously, it had been difficult to see any bitter or aversive taste reinforced memory scores 24 hours post-training in flies (Das et al., 2014). Based on above results, it is possible that T-maze conditioning protocols that do not normally yield scores at a certain time point, may do so with $Y$-maze testing. We therefore verified whether assaying aversive taste learning in $\mathrm{Y}$-mazes could enable the study of long-term taste aversion.

Copper sulphate $\left(\mathrm{CuSO}_{4}\right)$, a compound listed as a pesticide, is toxic to flies (Živanov-Čurlis et al., 2006; Alaraby, Hernández and Marcos, 2017; Balinski and Woodruff, 2017; Halmenschelager and da Rocha, 2019), and our unpublished data suggests that flies avoid it. Therefore, we decided to condition $\sim 24$ hour starved flies both in T-maze tubes and in training vials with $80 \mathrm{mM} \mathrm{CuSO}_{4}$ along with $200 \mathrm{mM}$ arabinose, a sweet but not metabolisable sugar, as a carrier to promote ingestion of $\mathrm{CuSO}_{4}$. Parallelly, flies were also conditioned with $200 \mathrm{mM}$ arabinose only. 24 hours after training, T-maze trained flies were tested in T-mazes (TT) and vial-trained flies were tested in Y-mazes (VY).

We saw that training and testing in the T-maze yielded a low positive score with 200 $\mathrm{mM}$ arabinose and a low aversive score with $80 \mathrm{mM} \mathrm{CuSO}_{4}+200 \mathrm{mM}$ arabinose (Fig 4A). Previous work suggests that when mixed appetitive and aversive stimuli are used for training, the memory score is the sum of the parallel and opposing memories from the two components (Das et al., 2014). Therefore, the difference between the means of the two groups (arabinose and $\mathrm{CuSO}_{4}+$ arabinose ) is a measure of the aversiveness of $\mathrm{CuSO}_{4}$. This difference is modest in the case of the TT groups and makes it difficult to further investigate (Fig 4A). In contrast, with the VY groups, both the approach scores with $200 \mathrm{mM}$ arabinose and the avoidance scores with $80 \mathrm{mM} \mathrm{CuSO}_{4}+200 \mathrm{mM}$ arabinose are greater and the difference between their means substantial (Fig 4A). We 
concluded that $\mathrm{Y}$-maze testing can enhance aversive memory scores too, making it possible to study the underlying neural mechanisms.

Because we could see a robust $\mathrm{CuSO}_{4}$ reinforced 24-hour aversive memory, we decided to investigate whether such memory is protein synthesis-dependent using Y-mazes. For this, experimental flies were kept on $35 \mathrm{mM}$ of cycloheximide, a protein synthesis inhibitor, mixed in $0.75 \%$ agar during the $\sim 17$ hour starvation period before training. Control flies were not exposed to cycloheximide. As before, flies were conditioned with $200 \mathrm{mM}$ arabinose or $80 \mathrm{mM} \mathrm{CuSO}_{4}+200 \mathrm{mM}$ arabinose and tested for 24-hour memory. They were briefly fed, for 2 minutes on 2 M sucrose, 6 hours after training to keep enough flies alive in the wake of cycloheximide exposure. We observed that both $200 \mathrm{mM}$ arabinose memory and $80 \mathrm{mM} \mathrm{CuSO}_{4}+200 \mathrm{mM}$ arabinose reinforced memories were reduced in the CXM fed (CXM+) group compared to the control (CXM-) group. We concluded that formation of both arabinose reinforced appetitive and $\mathrm{CuSO}_{4}$ reinforced aversive memories were sensitive to CXM treatment and thus likely to be protein synthesis dependent (Fig 4B). 


\section{Figure 4}

bioRxiv preprint doi: https://doi.org/10.1101/2020.11.18.386128; this version posted November 20, 2020. The copyright holder for this preprint (which was not certified by peer review) is the author/funder, who has granted bioRxiv a license to display the preprint in perpetuity. It is made A

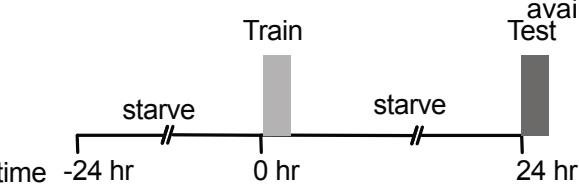
available under aCC-BY-NC-ND 4.0 International license.

time
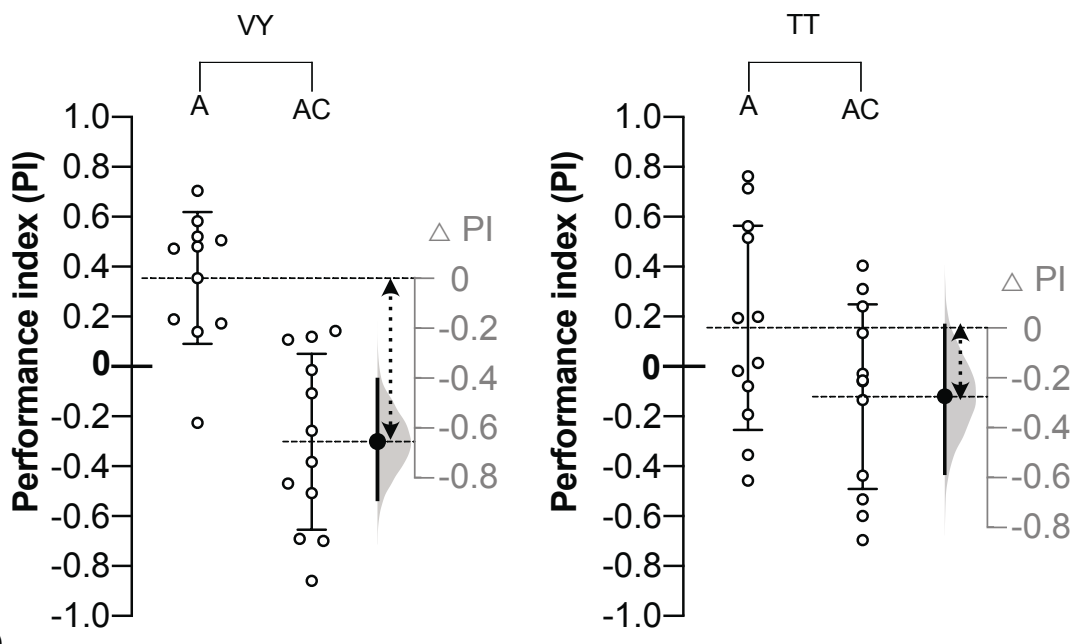

B

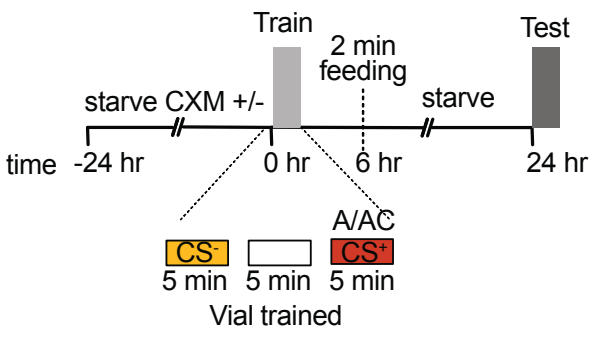

$200 \mathrm{mM}$ arabinose-agar (A) OR

$80 \mathrm{mM}$ CuSO $4+200 \mathrm{mM}$ arabinose-agar (AC)

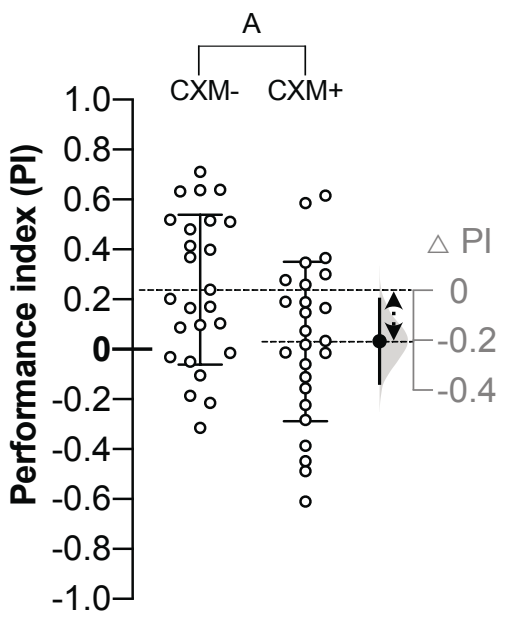

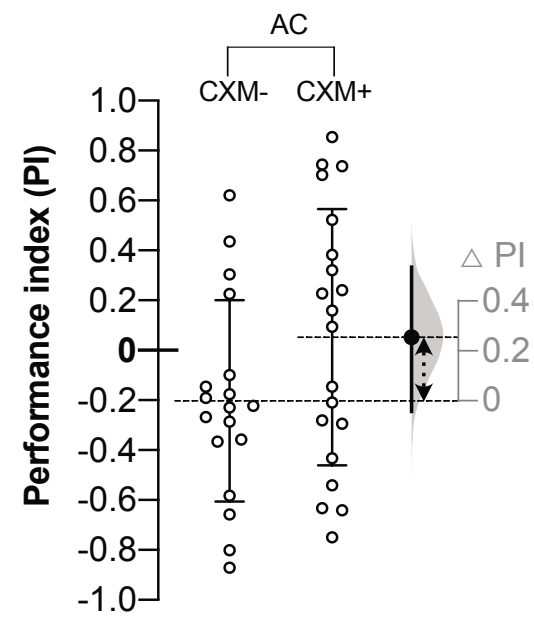


Figure 4: Testing in Y-mazes enables studying of novel memory phenotypes: testing protein synthesis dependence of sweet and aversive taste reinforced memories. (A) Enhanced $\mathrm{CuSO}_{4}$ reinforced aversive memory is seen in the $\mathrm{Y}$-maze tested groups (VY) compared to the T-maze tested group (TT). In the Y-maze tested groups, the difference in memory performance between $200 \mathrm{mM}$ arabinose training (VY-A; $n=12 ; C^{+} O C T=6, M C H=6$ ) and the $80 \mathrm{mM} \mathrm{CuSO}_{4}+200 \mathrm{mM}$ arabinose training (VY-AC; $n=12 ; \mathrm{CS}^{+} \mathrm{OCT}=6, \mathrm{MCH}=6$ ) was substantial. In contrast when the same experiment was done with T-mazes, memory performance difference between $200 \mathrm{mM}$ arabinose training (TT-A; $n=11 ; \mathrm{CS}^{+} \mathrm{OCT}=5, \mathrm{MCH}=6$ ) and $80 \mathrm{mM} \mathrm{CuSO}_{4}+200 \mathrm{mM}$ arabinose training (TT-AC; $n=12 ; C S^{+} O C T=6, M C H=6$ ) was modest. (B) To ascertain protein synthesis dependence of robust aversive taste memories, flies were trained in vials and tested for 24-hour memory performance in Y-mazes. $200 \mathrm{mM}$ arabinose reinforced appetitive memory performance is reduced upon cycloheximide treatment $\left(\mathrm{A}-\mathrm{CXM}+; n=25 ; \mathrm{CS}^{+} \mathrm{OCT}=12, \mathrm{MCH}=13\right.$ ) compared to the control (A-CXM-; $n=25 ; \mathrm{CS}^{+}$ $O C T=12, M C H=13)$. Further, $80 \mathrm{mM} \mathrm{CuSO}_{4}+200 \mathrm{mM}$ arabinose reinforced aversive memory performance upon cycloheximide treatment $\left(\mathrm{AC}-\mathrm{CXM}+; n=18 ; C S^{+} O C T=9\right.$, $M C H=9$ ) was also reduced compared to the control (AC-CXM-; $n=20 ; C S^{+} O C T=10$, $\mathrm{MCH}=10$ ). To increase survival, all flies were briefly fed for 2 minute on $2 \mathrm{M}$ sucrose, 6 hours after training. To the right of each graph, effect size or the difference in mean performance index $(\Delta \mathrm{PI})$ plots are shown. These plots depict the bootstrap sampling error distribution curve of $\Delta \mathrm{PI}$. The actual mean difference of the groups is depicted as a large black dot and the $95 \% \mathrm{Cl}$ is indicated by the ends of the vertical error bars. Double-headed dashed arrows between the group means indicate the groups being compared. 


\section{Discussion}

\section{Y-maze designs for olfactory behaviours}

Effective study of neural circuitry requires robust behavioural assays. It was the development of reliable learning and memory assays that enabled the neurogenetic study of fly learning and memory (Quinn, Harris and Benzer, 1974; Tully et al., 1994; Pitman et al., 2009; Tabone Christopher and de Belle, 2014). The olfactory conditioning and testing assembly we have described here has the potential to uncover novel memory phenotypes by virtue of the superior scores it generates. The Y-mazes are easy to build and costs a fraction of other conditioning setups currently in use. The lack of vacuum-assisted odour streams does away with the need of tubing, clamps, vacuum pumps and airflow monitoring, making it much simpler to handle.

Y-maze setups allowing free movement are common in rodent studies to measure spatial working memory and exploratory behaviour (Kraeuter, Guest and Sarnyai, 2019). Miniature single fly Y-mazes have been used in flies for the same purpose (Buchanan, Kain and de Bivort, 2015; Lewis et al., 2017; Cleal et al., 2020). Y-mazes have also been used for naive and learned odour preferences in insects other than flies (Dupuy et al., 2006; Carcaud et al., 2009; Josens, Eschbach and Giurfa, 2009). One of the first conditioning studies with Drosophila included Y-maze based visual conditioning experiments. Flies were trained in one arm of the $\mathrm{Y}$-maze to avoid a specific wavelength of light paired with aversive quinine taste. However, scores in this visual discrimination assay were quite low (Quinn, Harris and Benzer, 1974). Y-mazes have also been used recently for testing learned odour preference of flies previously conditioned with sugar or alcohol (Kaun et al., 2011; Albin et al., 2015; Nunez, Azanchi and Kaun, 2018). In these designs active and competing odour flows were generated in the arms of the Y-mazes for flies to choose between the conditioned odour stimuli. T-mazes and more often Y-mazes have been used as 'olfactometers' to measure olfactory choice, acuity and habituation in flies (Rodrigues and Siddiqi, 1978; Chakraborty, Goswami and 
Siddiqi, 2009; Das et al., 2011; Simoes, Ott and Niven, 2011; Simonnet, Berthelot-Grosjean and Grosjean, 2014).

\section{Y-mazes against T-mazes}

We have compared our Y-maze assembly directly to the T-maze design refined in (Tully and Quinn, 1985). Our appetitive and aversive gustatory cue reinforced memory scores from the Y-mazes were markedly greater than from the T-mazes (Fig 4). We also report durable appetitive memory scores up to 7 days in the Y-mazes. In contrast, T-maze PI scores were lower than 0.1 by day 5 in our hands (Fig 3). This suggests that memory performance will most likely be non-existent by day 7 in the T-maze. This trend is in agreement with earlier studies where training was done with dried sugar (as opposed to sugar in agar here) and tested in conventional T-mazes (Krashes and Waddell, 2008; Colomb et al., 2009). Going by the 7-day score with Y-mazes, flies are likely to exhibit memory performance even at later time points. Previously, such long-lasting appetitive scores from testing in T-mazes, have only been reported upon artificial induction of appetitive memory in flies (Huetteroth et al., 2015).

Our experiments suggest that testing in the Y-mazes is key to the improved scores. We speculate that this improvement over T-mazes is due to some or all of the following possibilities; a) an increased path length from the end of the cut pipette tips to the Y-junction and hence an extended odour gradient, b) increased time for choice, coupled with factors outlined in (a), might allow for a more reflective than reflexive choice, c) The trapping mechanism that prevent change in preference once choice is made d) reduced crowding at the choice point, preventing a 'stampede' like effect (Quinn, Harris and Benzer, 1974) and e) lack of airflow in the setup could help increase correct choice as flies do not have to struggle against fast odour streams. 
We also demonstrate that, like in T-mazes, memory formation in our setup depends on dopamine signaling to the mushroom body neurons. We did not directly test whether mushroom body neuronal activity is required for memory retrieval in the Y-mazes.

Typically, when using T-mazes, scores from two fly groups reciprocally trained with the two odours and tested in the same T-maze, are averaged to compute a single data point. This is done to negate any intrinsic odour bias in the specific T-maze machine used. However, as no single $\mathrm{Y}$-maze was reused for testing, instead of averaging reciprocal odour-trained groups, all Y-maze tested groups were represented as individual data points. Further, to maintain parity we also adapted a similar representation for T-maze data in all experiments here. Importantly, not averaging the memory scores from pairs of reciprocally trained groups, the mean of the data population is not altered. In fact, averaging decreases the population standard deviation. We do maintain an equal number of groups conditioned with each odour to balance out odour biases in experiments.

\section{The $\mathrm{Y}$-mazes are versatile}

Testing in Y-mazes can be potentially coupled to other training protocols. For example, electric shock conditioned flies can be tested in Y-mazes to improve test scores. Y-mazes are compatible with common genetic manipulation techniques used in flies. We demonstrate its use with thermogenetic silencing of neurons. It is easy to carry out both training and testing at $32^{\circ} \mathrm{C}$ by simply placing the training vials and the $\mathrm{Y}$-mazes inside a chamber of appropriate temperature (Fig S1F). Care should be taken to balance odour concentrations at the testing temperature to ensure equal distribution of naive flies. With slight modifications, the $\mathrm{Y}$-mazes should be optogenetic compatible too. As is, training is done in transparent vials. The testing setup can be made optogenetics-ready by using transparent Y-connectors. The Y-mazes described here do not require a vacuum source to work, making them readily portable and can be easily used for science outreach outside the laboratory. 
A perceived drawback of our assay is that it is fully manual. Training, testing and counting flies are not automated. But the setup is simple enough to be amenable to mechanization. Another drawback could be the number of flies that are used ( 50-100 for $n=1)$. While the strength of group-based memory assays is in the numbers, one can easily shrink the dimensions of the connectors to fit with smaller tubes and perform the assay with 25-50 flies. Assaying memory with even smaller group sizes may increase the variability of the data as choice of individual flies will have a greater influence on the score.

\section{Revealing novel memory phenotypes with the Y-mazes}

When flies are odour-conditioned for a single round with heat or bitter tasting compounds, the memories formed decay within a few hours (Quinn, Harris and Benzer, 1974; Tully and Quinn, 1985; Das et al., 2014; Galili et al., 2014; Masek et al., 2015). Single round of shock reinforced memories also decays within a few hours or show relatively weak 24-hour memory performance, mainly depending on the voltage, protocol and setup used (Quinn, Harris and Benzer, 1974; Tully and Quinn, 1985; Tully et al., 1994). Robust 24-hour aversive memory reinforced by shock stimulus requires multiple rounds of massed or spaced training (Tully et al., 1994). However, no such effect is reported for training with heat, bitter taste or other aversive stimuli. Our improved 24-hour appetitive memory scores with the Y-mazes encouraged us to test whether robust taste reinforced aversive memories can be assayed. In keeping with our appetitive memory scores, here we report the presence of a robust 24-hour aversive taste memory reinforced by $\mathrm{CuSO}_{4}$. This then prompted us to test whether this long-lasting aversive memory was protein synthesis-dependent.

Protein-synthesis dependence, often tested by the use of pharmacological blockers of protein synthesis, has been considered a hallmark of 'long-term memory' (LTM) in flies and other species (Davis and Squire, 1984; Hernandez and Abel, 2008). Memory 
formed by single session shock training is not sensitive to the feeding of the protein synthesis inhibitor cycloheximide, unless flies are starved before training and fed immediately afterwards (Tully et al., 1994; Hirano et al., 2013; Plaçais and Preat, 2013). Formation of protein synthesis dependent shock-reinforced aversive memories are also seen upon multiple session spaced training, but not upon massed training (Tully et al., 1994).

A recent study in flies highlighted the importance of the order of $\mathrm{CS}^{+}$odour-shock association during spaced training. They showed that when $\mathrm{CS}^{+}$precedes $\mathrm{CS}^{-}$, both a $\mathrm{CS}^{+}$linked aversive memory and a CS' linked appetitive 'safety' memory is formed. Thus the 24-hour memory performance, showing aversion to the $\mathrm{CS}^{+}$odour, is ultimately a summation of both these memories (Jacob and Waddell, 2020). Interestingly, only the CS- linked 24-hour appetitive safety memory, not the $\mathrm{CS}^{+}$linked aversive memory, is protein synthesis dependent. Thus adding clarity to why spaced trained flies show reduced aversive memory performance upon cycloheximide exposure (Tully et al., 1994). In contrast, when CS- precedes CS $^{+}$during spaced shock training, no parallel safety memory is formed (Jacob and Waddell, 2020).

Incidentally, protein synthesis independent, shock reinforced aversive memory, for 14 days and possibly more, have been reported in flies. This memory, called context dependent LTM (cLTM), is retrieved only when the context of copper wires that deliver shock during training is presented during testing inside the T-maze choice tubes. However, while the formation of CLTM is dopamine dependent, its retrieval is mediated through the lateral horn neurons (Zhao et al., 2019).

In our experiments, we found that the aversive memory formed by a single 5 minute session of $80 \mathrm{mM} \mathrm{CuSO}_{4}$ (with $200 \mathrm{mM}$ arabinose carrier) training, is severely impaired when flies were exposed to the protein-synthesis inhibitor cycloheximide. This was also the case for memory reinforced by $200 \mathrm{mM}$ arabinose alone. Since neither aversive nor 
sweet taste reinforced 24-hour memories have been observed robustly before, their protein synthesis dependence had not been tested. Previous studies suggest that starvation biases the brain away from protein synthesis dependent LTM formation. If flies are food replete or have been fed proximally enough to when they are conditioned with shock-they form LTM (Hirano et al., 2013; Plaçais and Preat, 2013). Similarly, feeding after 3 hours of conditioning with sweet only sugar aids in the formation of LTM. However, no LTM is formed when flies are fed after 6 hours of conditioning with sweet only sugar (Musso, Tchenio and Preat, 2015). In our case flies were starved before training, kept starved after training, but they were briefly fed for 2 minutes with $2 \mathrm{M}$ sucrose, 6 hours after training. We cannot rule out that this brief feeding might induce LTM formation without thorough investigation. Nonetheless, our results imply that either a) gustatory reinforcement forms LTM independently of energy status or b) LTM formation can be induced with a brief dose of feeding even 6 hours after training. Our ongoing studies are pursuing these and other related questions regarding the nature of long lasting aversive memories using the Y-mazes.

In conclusion, we have highlighted the potential benefits of a newly designed Y-maze assembly, the most important of which is improved memory scores. Potential users can also easily modify parts of the assembly to suit their needs. We anticipate that the Y-maze assembly described here will be widely adopted by researchers investigating mechanisms of learning and memory in flies. 


\section{Acknowledgements}

We would like to thank all our colleagues in the brain and feeding behaviour laboratory, NCCS, for their support, help with experiments and for providing feedback on the manuscript. We also thank Suewei Lin, Vincent Croset, Emamanuel Perisse, Johannes Felsenberg, Wolf Huetteroth and Scott Waddell for their critical reading of the manuscript and providing valuable feedback and insights. We thank Paola Cognini- our vial training paradigm has been inspired by her similar design. We thank Rajkumar Pawar for lab ordering, making fly food, stock maintenance and Abhay Jadhav for washing fly bottles and vials. RM is funded by a PhD fellowship from NCCS. MRT is funded by a Department of Biotechnology (DBT) doctoral scholarship. FMI and MS were funded from a Ramanujan Fellowship to GD. GD is a Ramanujan Fellow (SB/S2/RJN-048/2017) awarded by the Science and Engineering Research Board (SERB), part of the Department of Science \& Technology (DST), Government of India. The lab is also funded by a Core Research Grant (CRG/2019/005587) from SERB and by generous intramural funding support from NCCS.

\section{Contributions}

G.D. and R.M. conceived the design of this particular Y-maze. Initial trials and design refinement was done by R.M., M.S. and G.D. All experiments described here were performed by R.M., F.M.I and M.T. G.D. directed the research and wrote the manuscript with inputs from R.M.

\section{Competing interests}

The authors declare no competing interests.

\section{Data availability}

All raw data, including STL files for the Y-maze parts are available upon request to the corresponding author. 


\section{REFERENCES}

Alaraby, M., Hernández, A. and Marcos, R. (2017) 'Copper oxide nanoparticles and copper sulphate act as antigenotoxic agents in drosophila melanogaster', Environmental and molecular mutagenesis, 58(1), pp. 46-55.

Albin, S. D. et al. (2015) 'A Subset of Serotonergic Neurons Evokes Hunger in Adult Drosophila', Current biology: CB, 25(18), pp. 2435-2440.

Balinski, M. A. and Woodruff, R. C. (2017) 'Differential sexual survival of Drosophila melanogaster on copper sulfate', Genetica, 145(2), pp. 131-137.

Boto, T., Stahl, A. and Tomchik, S. M. (2020) 'Cellular and circuit mechanisms of olfactory associative learning in Drosophila', Journal of neurogenetics, 34(1), pp. 36-46.

Buchanan, S. M., Kain, J. S. and de Bivort, B. L. (2015) 'Neuronal control of locomotor handedness in Drosophila', Proceedings of the National Academy of Sciences of the United States of America, 112(21), pp. 6700-6705.

Burke, C. J. et al. (2012) 'Layered reward signalling through octopamine and dopamine in Drosophila', Nature, 492(7429), pp. 433-437.

Carcaud, J. et al. (2009) 'Odour aversion after olfactory conditioning of the sting extension reflex in honeybees', The Journal of experimental biology, 212(Pt 5), pp. $620-626$.

Chakraborty, T. S., Goswami, S. P. and Siddiqi, O. (2009) 'Sensory correlates of imaginal conditioning in Drosophila melanogaster', Journal of neurogenetics, 23(1-2), pp. 210-219.

Cleal, M. et al. (2020) 'The Free-movement pattern Y-maze: A cross-species measure of working memory and executive function', Behavior research methods. doi: 10.3758/s13428-020-01452-x.

Cognigni, P., Felsenberg, J. and Waddell, S. (2018) 'Do the right thing: neural network mechanisms of memory formation, expression and update in Drosophila', Current opinion in neurobiology, 49, pp. 51-58.

Colomb, J. et al. (2009) 'Parametric and genetic analysis of Drosophila appetitive long-term memory and sugar motivation', Genes, brain, and behavior, 8(4), pp. 407-415.

Das, G. et al. (2014) 'Drosophila learn opposing components of a compound food stimulus', Current biology: CB, 24(15), pp. 1723-1730.

Das, S. et al. (2011) 'Plasticity of local GABAergic interneurons drives olfactory 
habituation', Proceedings of the National Academy of Sciences of the United States of America, 108(36), pp. E646-54.

Davis, H. P. and Squire, L. R. (1984) 'Protein synthesis and memory: a review', Psychological bulletin, 96(3), pp. 518-559.

Dudai, Y. (1983) 'Mutations affect storage and use of memory differentially in Drosophila', Proceedings of the National Academy of Sciences of the United States of America, 80(17), pp. 5445-5448.

Dupuy, F. et al. (2006) 'Individual olfactory learning in Camponotus ants', Animal behaviour, 72(5), pp. 1081-1091.

Galili, D. S. et al. (2014) 'Converging circuits mediate temperature and shock aversive olfactory conditioning in Drosophila', Current biology: CB, 24(15), pp. 1712-1722.

Griffith, L. C. (2012) 'Identifying behavioral circuits in Drosophila melanogaster: moving targets in a flying insect', Current opinion in neurobiology, 22(4), pp. 609-614.

Halmenschelager, P. T. and da Rocha, J. B. T. (2019) 'Biochemical CuSO4 Toxicity in Drosophila melanogaster Depends on Sex and Developmental Stage of Exposure', Biological trace element research, 189(2), pp. 574-585.

Harris, W. A. (2008) 'Seymour Benzer 1921-2007 The Man Who Took Us from Genes to Behaviour', PLoS biology, 6(2), p. e41.

Hernandez, P. J. and Abel, T. (2008) 'The role of protein synthesis in memory consolidation: progress amid decades of debate', Neurobiology of learning and memory, 89(3), pp. 293-311.

Hirano, Y. et al. (2013) 'Fasting launches CRTC to facilitate long-term memory formation in Drosophila', Science, 339(6118), pp. 443-446.

Ho, J. et al. (2019) 'Moving beyond P values: data analysis with estimation graphics', Nature methods, 16(7), pp. 565-566.

Huetteroth, W. et al. (2015) 'Sweet taste and nutrient value subdivide rewarding dopaminergic neurons in Drosophila', Current biology: $C B, 25(6)$, pp. 751-758.

Ichinose, T. and Tanimoto, H. (2016) 'Dynamics of memory-guided choice behavior in Drosophila', Proceedings of the Japan Academy. Series B, Physical and biological sciences, 92(8), pp. 346-357.

Jacob, P. F. and Waddell, S. (2020) 'Spaced Training Forms Complementary Long-Term Memories of Opposite Valence in Drosophila', Neuron, 106(6), pp. 977-991.e4. 
Jenett, A. et al. (2012) 'A GAL4-driver line resource for Drosophila neurobiology', Cell reports, 2(4), pp. 991-1001.

Josens, R., Eschbach, C. and Giurfa, M. (2009) 'Differential conditioning and long-term olfactory memory in individual Camponotus fellah ants', The Journal of experimental biology, 212(Pt 12), pp. 1904-1911.

Kahsai, L. and Zars, T. (2011) 'Learning and memory in Drosophila: behavior, genetics, and neural systems', International review of neurobiology, 99, pp. 139-167.

Kaun, K. R. et al. (2011) 'A Drosophila model for alcohol reward', Nature neuroscience. doi: 10.1038/nn.2805.

Keene, A. C. et al. (2006) 'Drosophila Dorsal Paired Medial Neurons Provide a General Mechanism for Memory Consolidation', Current biology: CB, 16(15), pp. 1524-1530.

Keene, A. C. and Waddell, S. (2007) 'Drosophila olfactory memory: single genes to complex neural circuits', Nature reviews. Neuroscience, 8(5), pp. 341-354.

Kim, Y.-C., Lee, H.-G. and Han, K.-A. (2007) 'Classical reward conditioning in Drosophila melanogaster', Genes, brain, and behavior, 6(2), pp. 201-207.

Kim, Y.-C., Lee, H.-G. and Han, K.-A. (2007) 'D1 dopamine receptor dDA1 is required in the mushroom body neurons for aversive and appetitive learning in Drosophila', The Journal of neuroscience: the official journal of the Society for Neuroscience, 27(29), pp. 7640-7647.

Kitamoto, T. (2001) 'Conditional modification of behavior in Drosophila by targeted expression of a temperature-sensitive shibire allele in defined neurons', Journal of neurobiology, 47(2), pp. 81-92.

Kraeuter, A.-K., Guest, P. C. and Sarnyai, Z. (2019) 'The Y-Maze for Assessment of Spatial Working and Reference Memory in Mice', Methods in molecular biology , 1916, pp. 105-111.

Krashes, M. J. and Waddell, S. (2008) 'Rapid consolidation to a radish and protein synthesis-dependent long-term memory after single-session appetitive olfactory conditioning in Drosophila', The Journal of neuroscience: the official journal of the Society for Neuroscience, 28(12), pp. 3103-3113.

Krashes, M. J. and Waddell, S. (2011) 'Drosophila appetitive olfactory conditioning', Cold Spring Harbor protocols, 2011(5), p. db.prot5609.

Krashes, M. J. and Waddell, S. (2011) 'Drosophila aversive olfactory conditioning', Cold Spring Harbor protocols. Available at: 
http://cshprotocols.cshlp.org/content/2011/5/pdb.prot5608.short.

Lewis, S. A. et al. (2017) 'Spontaneous alternation: A potential gateway to spatial working memory in Drosophila', Neurobiology of learning and memory, 142(Pt B), pp. 230-235.

Lin, S. et al. (2014) 'Neural correlates of water reward in thirsty Drosophila', Nature neuroscience, 17(11), pp. 1536-1542.

Liu, C. et al. (2012) 'A subset of dopamine neurons signals reward for odour memory in Drosophila', Nature, 488(7412), pp. 512-516.

Masek, P. et al. (2015) 'A dopamine-modulated neural circuit regulating aversive taste memory in Drosophila', Current biology: CB, 25(11), pp. 1535-1541.

McGuire, S. E., Deshazer, M. and Davis, R. L. (2005) 'Thirty years of olfactory learning and memory research in Drosophila melanogaster', Progress in neurobiology, 76(5), pp. 328-347.

Musso, P.-Y., Tchenio, P. and Preat, T. (2015) 'Delayed dopamine signaling of energy level builds appetitive long-term memory in Drosophila', Cell reports, 10(7), pp. 1023-1031.

Nunez, K. M., Azanchi, R. and Kaun, K. R. (2018) 'Cue-Induced Ethanol Seeking in Drosophila melanogaster Is Dose-Dependent', Frontiers in physiology, 9, p. 438.

Owald, D., Lin, S. and Waddell, S. (2015) 'Light, heat, action: neural control of fruit fly behaviour', Philosophical transactions of the Royal Society of London. Series B, Biological sciences, 370(1677), p. 20140211.

Pfeiffer, B. D. et al. (2008) 'Tools for neuroanatomy and neurogenetics in Drosophila', Proceedings of the National Academy of Sciences of the United States of America, 105(28), pp. 9715-9720.

Pfeiffer, B. D. et al. (2010) 'Refinement of tools for targeted gene expression in Drosophila', Genetics, 186(2), pp. 735-755.

Pipkin, J. (2020) 'Mapping the mind of a fly', eLife, 9. doi: 10.7554/eLife.62451.

Pitman, J. L. et al. (2009) 'There are many ways to train a fly', Fly, 3(1), pp. 3-9.

Plaçais, P.-Y. and Preat, T. (2013) 'To favor survival under food shortage, the brain disables costly memory’, Science, 339(6118), pp. 440-442.

Quinn, W. G., Harris, W. A. and Benzer, S. (1974) 'Conditioned behavior in Drosophila melanogaster', Proceedings of the National Academy of Sciences of the United States 
of America, 71(3), pp. 708-712.

Rodrigues, V. and Siddiqi, O. (1978) 'Genetic analysis of chemosensory pathway', Proceedings of the Indian Academy of Sciences - Section B, 87(7), pp. 147-160.

Scaplen, K. M. et al. (2020) 'Circuits that encode and guide alcohol-associated preference', eLife, 9. doi: 10.7554/eLife.48730.

Scheffer, L. K. et al. (2020) 'A connectome and analysis of the adult Drosophila central brain', eLife, 9. doi: 10.7554/eLife.57443.

Schwaerzel, M. et al. (2003) 'Dopamine and octopamine differentiate between aversive and appetitive olfactory memories in Drosophila', The Journal of neuroscience: the official journal of the Society for Neuroscience, 23(33), pp. 10495-10502.

Shohat-Ophir, G. et al. (2012) 'Sexual deprivation increases ethanol intake in Drosophila', Science, 335(6074), pp. 1351-1355.

Simoes, P., Ott, S. R. and Niven, J. E. (2011) 'Associative olfactory learning in the desert locust, Schistocerca gregaria', Journal of Experimental Biology, pp. 2495-2503. doi: 10.1242/jeb.055806.

Simonnet, M. M., Berthelot-Grosjean, M. and Grosjean, Y. (2014) 'Testing Drosophila olfaction with a Y-maze assay', Journal of visualized experiments: JoVE, (88). doi: 10.3791/51241.

Simpson, J. H. (2009) 'Chapter 3 Mapping and Manipulating Neural Circuits in the Fly Brain', in Advances in Genetics. Academic Press, pp. 79-143.

Simpson, J. H. and Looger, L. L. (2018) 'Functional Imaging and Optogenetics in Drosophila', Genetics, 208(4), pp. 1291-1309.

Skoulakis, E. M. C. and Grammenoudi, S. (2006) 'Dunces and da Vincis: the genetics of learning and memory in Drosophila', Cellular and molecular life sciences: CMLS, 63(9), pp. 975-988.

Tabone Christopher, J. and de Belle, J. S. (2014) 'Olfactory learning and memory assays', in Behavioral Genetics of the Fly (Drosophila Melanogaster). Cambridge University Press, pp. 231-249.

Tempel, B. L. et al. (1983) 'Reward learning in normal and mutant Drosophila', Proceedings of the National Academy of Sciences of the United States of America, 80(5), pp. 1482-1486.

Tully, T. et al. (1994) 'Genetic dissection of consolidated memory in Drosophila', Cell, 79(1), pp. 35-47. 
Tully, T. and Quinn, W. G. (1985) 'Classical conditioning and retention in normal and mutant Drosophila melanogaster', Journal of comparative physiology. A, Sensory, neural, and behavioral physiology, 157(2), pp. 263-277.

Vosshall, L. B. (2007) 'Into the mind of a fly’, Nature, 450(7167), pp. 193-197.

White, B. H. (2016) 'What genetic model organisms offer the study of behavior and neural circuits', Journal of neurogenetics, 30(2), pp. 54-61.

White, B. H. and Peabody, N. C. (2009) 'Neurotrapping: cellular screens to identify the neural substrates of behavior in Drosophila', Frontiers in molecular neuroscience, 2, p. 20.

Yamagata, N. et al. (2015) 'Distinct dopamine neurons mediate reward signals for shortand long-term memories', Proceedings of the National Academy of Sciences of the United States of America, 112(2), pp. 578-583.

Yoshihara, M. and Ito, K. (2012) 'Acute genetic manipulation of neuronal activity for the functional dissection of neural circuits-a dream come true for the pioneers of behavioral genetics', Journal of neurogenetics, 26(1), pp. 43-52.

Zhao, B. et al. (2019) 'Long-term memory is formed immediately without the need for protein synthesis-dependent consolidation in Drosophila', Nature communications, 10(1), p. 4550.

Zheng, Z. et al. (2018) 'A Complete Electron Microscopy Volume of the Brain of Adult Drosophila melanogaster', Cell, 174(3), pp. 730-743.e22.

Živanov-Čurlis, J. et al. (2006) 'The Influence of Long-Term Intake of Copper and Chromium Compounds on Reproductive Ability and Development of Drosophila Melanogaster', Biotechnology, biotechnological equipment, 20(2), pp. 62-66. 